\title{
Effect of Active Tea Extract on Sports Ability
}

\author{
Junbiao Kang \\ School of Physical Education, Zhengzhou Institute of Technology, Zhengzhou 450000, China \\ tuke126@126.com \\ Corresponding Author: Junbiao Kang
}

Keywords: Active Tea Extract; Sports Ability.

\begin{abstract}
A large number of facts have proved that active tea extract is beneficial to human health and can prevent certain disease, and it of course also have a certain impact on the sports ability of athletes, which is mainly because the active tea extract has a unique effect on improving physiological functions, regulating nerves, inhibiting and sterilizing bacteria etc. Active tea extract can refresh the mind and relieve the fatigue for people, especially for athletes. A couple of tea during training or competition can boost athletes' spirit and enhance the feeling ability and reaction ability of motor nerve stimulation of athletes, which will help to improve the quality of training and competition results. In this paper, after having a series of analysis and demonstration, the effects of active tea extract on the athlete's athletic ability and their performance are further analyzed.
\end{abstract}

\section{Introduction}

Tea is one of the most popular drinks in China, with attractive aroma and unique taste, as well as a high value of health care. Modern epidemiological studies have shown that drinking tea have an effect on stimulating nerve center and eliminating fatigue, along with decanting, detoxification and diuretic effects and so on. New research progress shows that the active ingredients of tea also play roles in anti-oxidation, anti-mutation and anti-cancer. Active tea extract mainly include alkaloids, tea polyphenols, carbohydrates, proteins, amino acids, vitamins and trace elements an other nutrients needed by humans.

\section{Effects of active tea extract on physical fitness of athletes}

Regarding the effects of active tea extract on the sports ability of athletes, based on the inheritance and development of ancient views, combined with modern medical technology, some new ideas have been put forward, which are mainly reflected from the following several aspects:

\subsection{Refreshing people's mind and reducing fatigue}

The active tea extract has a refreshing effect, which is not affected by other factors. Meanwhile, drinking tea can improve the body's metabolism, blood outflow, gastric secretion, etc. However, drinking too much will cause central nervous anesthesia poisoning, which is harmful to human health. In the daily training of athletes, a cup of tea can stimulate the brain central nervous to make it excited, so as to achieve the purpose of refreshing athlete's mind and reducing fatigue.

\subsection{Enhancing renal excretion function}

The comparison of active ingredients in tea shows that the activity of active tea extract is stronger than that of caffeine, and the activity of caffeine is stronger than that of theobromine. Clinical trial results suggest that the active tea extract has the strongest diuretic effect, but the diuretic of theobromine is the most lasting. The main function of these components is to inhibit the re-absorption of renal tubule so as to increase the content of sodium and nitrogen ions in urine. Such compounds can excite the motor center of blood, directly relax the renal blood vessels, increase the renal blood flow and enhance the leaching rate of glomerulus. In addition, tea also contains quercetion and other flavonoids, saponins and aromatic compounds, etc., all of which have a strong diuretic effect. 


\subsection{Promoting the normal proceeding of metabolism}

Active tea extract is helpful to the food digestion mainly because it can enhance the peristalsis of the digestive tract and prevent the gastrointestinal disease. Active tea extract can affect the metabolism of fatty acid mainly because tea contains inositol, folic acid and other vitamin compounds, which can regulate the metabolism of fat. In addition to the promoting digestion function, tea can also treat the bleeding caused by gastrointestinal ulcer, which reason is that the polyphenolic compounds in tea can attach to the wound in the stomach under solid film state, thus playing a protective role. Moreover, tea also has the ability to absorb harmful substances to the human body, which not only can "purify" the microorganisms in digestive tract, but also can chemically cleanse the stomach, kidney and liver in a unique way.

\subsection{Protecting the eyes and nature the soul}

In active tea extract, there is a component called pro-vitamin A, which is commonly known as carotene. The active tea extract contains about 54.6 ug carotene, which is equivalent to about 91 international units of VA. Tea also contains another component called $\beta$-ionone, which is a precursor compound of VA and carotene biosynthesis. Carotene can prevent the dry eye disease formed by keratinocyte proliferation of epithelial tissues. Moreover, carotene can be converted into vitamin A in the body, and also can combine with protein to form rhodopsin in the retina to enhance the retinal photosensitivity, playing a role in the prevention and treatment of night blindness. Vitamin B2 in the tea can treat keratitis. Vitamin $\mathrm{C}$ extracted from the tea can increase the resistance, and also plays a role in the treatment of cataract lens opacity.

\subsection{Supplying vitamin $C$ to prevent scurvy}

In addition to the above mentioned ingredients, there is also a large number of vitamin $\mathrm{C}$ in the tea, especially the green tea. Each 100 g active green tea extract contains VC 180 mg. Lacking vitamin C in the body will lead to vascular permeability damage, causing petechial hemorrhages, tooth caries bleeding and serous cavity hemorrhage of muscle or joint capsule etc. At the same time, vitamin $\mathrm{C}$ deficiency will reduce the resistance, leading to the blocked wound healing, thus emerging the gum disease, friable tissues, which is prone to fracture periosteum split, and dental caries. Besides, the flavonoids in the active tea extract have an important synergistic effect on the treatment of scurvy and vitamin $\mathrm{C}$, and it is also helpful to the body's absorption of vitamin $\mathrm{C}$, to enhance the toughness of microvascular.

\section{Effects of active tea extract on the sports ability of athletes}

Since the 80s of last century, our country has began to attach importance to the sports ability of athletes. Many scholars have done some research in this area and put forward that "sports performance, sports ability and training level are 3 different concepts". Then they also proposed that "The ability possessed by athletes to participate in competitive games and create sports performance is called sports ability, which is a synthesis of athlete's physical ability, skill, intelligence and psychological ability". These theories make people gradually know that there are many factors that constitute the athlete's sports ability: the physical ability, skill, intelligence and psychological ability.

Many experts and scholars have put forward different opinions on the influence of athletes' sports ability. For example, "The sports ability of athletes is affected by factors such as shape, function, quality, skill, tactics, intelligence, psychology and so on,” Professor Tian Maijiu wrote in Sports Training (2010). Zhang Yingbo in "Sports Science" (2009) put forward that the seven factors affecting the athletic ability of athletes include the form, function, quality, skill, tactics, intelligence, psychology and so on. While some other studies on the athletes sports ability are directly pointing at specific indicators of various sports events and without distinguish between constituent elements and influencing factors.

In this case, we regard the form, function, quality, skills, tactics, intelligence and psychology as the factors that influence the athlete's sports ability, all of which seem to be similar from the method used in this research, but in terms of research ideas, the researches which regard these factors as constituent elements are directly directed at the athlete's own sports ability. Therefore, gray-box 
method should be used here. It should be clear that all the elements of sports ability and its internal structure should be seen as primary task, and try to move it closer to the white box so as to completely solve the assessment and diagnosis issues of sports ability. To make them as important influencing factors, the sports ability of athletes should only be studied indirectly with the black-box method. And the focus of these researches should be that how these factors affect the athletic ability, how it affects and how much it affects etc. Therefore, there is better to be a clear distinguish between constituents elements and influencing factors, which also involves the definition of the concept of "sports ability".

\subsection{Elements of athlete's sports ability}

In the study of the elements of the athlete's sports ability, "physical ability, skills, intelligence and psychological ability" were proposed initially. With the change of time and the deepening of research, it was expanded into "form, function, quality, skills, tactics, intelligence and psychology", which were collectively referred to as the 7 elements of sports ability model theory.

At present, the differences in the research of sports ability are mainly related to the relationship among the elements. The model of "sports ability barrel structure" proposed by Gen muyong, a Japanese scholar, argues that the relationship between the various components of sports ability is irreplaceable. An element in poor condition will form a bottleneck. To improve the sports ability, all elements must be in good condition, and the level of sports ability depends on the one of the worst factors.

However, in 1990s, Chinese scholar Liu Daqing put forward the "Unbalanced Structure Compensation Theory of Athlete's Sports Ability”, and thought that “The general model of athlete's sports ability reflects the commonness of things, showing the characteristics of equilibrium; the individual model of athlete's sports ability reflects the speciality of things, showing the characteristics of non-balance”. "In the athlete's sports ability constitution factors, the phenomenon of a poor quality or ability to compensate another highly developed quality or ability is called the sports ability unbalanced structure compensation". At the same time, they also thought that "the unbalanced structure compensation of athletes' sports ability is prevalent in the individual structure of athlete's athletic ability of different item groups, different levels and different periods", and pointed out that "There are many ways to compensate unbalanced structure of athlete's sports ability. According to the unbalanced structure and function of sports ability, it can be divided into unbalanced compensation and balanced compensation; according to the roles of advantageous ability played in the unbalanced structure of sports, it can be divided into amplification compensation and radiation compensation; according to the way of obtaining the superiority ability in the unbalanced structure of sports ability, it can be divided into exogenous compensation and endogenous compensation”.

From the practice point of view, the compensation theory of unbalanced structure of competitive ability is justified. Most of the front-line coaches deeply understand that because athletes often have their own merits, as well as weaknesses, which is particularly evident in projects requiring comprehensive capabilities. However, it's obvious that sports cloud-top ability unbalanced structure compensation theory is just a qualitative theoretical framework, which is still far away from practical training, especially there are some important issues which are still not clear such as what can compensate for what, or what is the mechanism and so on.

\subsection{Effects of tea extracts on sports ability of athletes}

In the current situation, on one hand, we will regard the sports ability as one of the factors which influence the athletic ability of athletes. Moreover, it has been confined to these 7 elements when we first began to study the subject. From 1990s to the present, the situations which put the "time" as one of the factors are significantly increased. For example, in the high-level tract race, Fu Qiuming's study on the time variation of pre-competition anxiety state of athletes and its influence on their performance, and the Xu Benli's study on the time rule of pre-competition state regulation of some elite athletes, as well as the study on space-time coordination theory of athlete competitive ability state transfer in physical power dominant fast power event group. Among them, there are more specific studies as well as macro-theoretical research. While the research on the influence of active tea extract on athlete's athletic ability is just beginning, this is a vital subject. Moreover, the results of 
this study have found that active tea extract indeed have the ability to improve the sports ability of athletes.

\section{Conclusion}

In conclusion, based on the theoretical research and practice demonstration, the results on the study of athlete's sports ability suggest that we can have a targeted arrangement of athletes training and diet, as well as drinking tea appropriatly. Therefore, we can start from two aspects. On one hand, from the perspective of sports training, aerobic and anaerobic endurance training all can reduce the athlete's power consumption, and reasonable arrangements for training can improve the body's ability to remove free radicals in the movement, which can effectively relieve muscular trauma in athletes. On the another hand, from the research on the active ingredients of tea extract, it has been found that active tea extract not only has the effect of scavenging free radicals, but has the effect of lowering the level of lipid peroxidation after exercise and reducing the generation of malonydialdehyde (MDA). Therefore, only by in-depth study and analysis of active tea extract on athletes sports ability, combined with reasonable and effective methods, the athlete's performance can be further improved and contribute to the development of sports.

\section{References}

[1] Zhang YB. The 6th Sports Science Conference. China Sport Science. 2010; 4.

[2] Liu DQ. The 5th National Sports Science Conference in 1997. China Sport Science. 2010; 1.

[3] Li S, Luo LY, Liu SJ et al. Effect of different concentrations of theaflavins and caffeine on the formation of tea cream. Science and Technology of Food Industry. 2015, 36(14):92-97.

[4] Yang L, Zhang G, Tao YJ et al. Interacting Effect of Polyphenols and Caffeine on Quality of Kuding Tea Drink. Guizhou Science. 2014, 32(5):66-70.

[5] Xu YQ, Liu P, Chen GS et al. Effect of Tea Polyphenols Concentration on the Stability of Green Tea Infusion. Journal of Tea Science. 2011, 31(6):525-531. 